\title{
La peine capitale aux États-Unis à la croisée de la fiction à substrat professionnel et de la source primaire : une étude comparative
}

\section{Sandrine Chapon}

\section{(2) OpenEdition}

Journals

Édition électronique

URL : http://journals.openedition.org/asp/2493

DOI : 10.4000/asp.2493

ISSN : 2108-6354

Éditeur

Groupe d'étude et de recherche en anglais de spécialité

Édition imprimée

Date de publication : 1 novembre 2011

Pagination : 21-39

ISSN : 1246-8185

Référence électronique

Sandrine Chapon, «La peine capitale aux États-Unis à la croisée de la fiction à substrat professionnel et de la source primaire : une étude comparative », ASp [En ligne], 60 | 2011, mis en ligne le 07 octobre 2014, consulté le 02 novembre 2020. URL : http://journals.openedition.org/asp/2493 ; DOI : https:// doi.org/10.4000/asp.2493

Ce document a été généré automatiquement le 2 novembre 2020.

Tous droits réservés 


\title{
La peine capitale aux États-Unis à la croisée de la fiction à substrat professionnel et de la source primaire : une étude comparative
}

\author{
Sandrine Chapon
}

1 Dans le domaine du droit, les enseignants des champs disciplinaires fondent l'acquisition des savoirs fondamentaux sur l'analyse de textes professionnels authentiques, arrêts de la Cour européenne des droits de l'homme, extraits du code civil, etc., alors que les enseignants de langue de spécialité utilisent plus volontiers l'article de presse ou la fiction. L'objectif de cet article est de comparer ces différents supports pédagogiques afin de déterminer si les ressources mises au service de l'objet enseigné constituent des documents pertinents pour l'apprentissage de la langue et culture de spécialité dans le secteur LANSAD. Le support télévisuel ou la coupure de presse relatifs à la même affaire sont-ils moins pertinents d'un point de vue de la terminologie que les textes primaires?

2 Nous proposons de fonder cette étude linguistique sur le thème de la peine de mort, l'un des éléments indispensables pour l'enseignant désireux de baliser les repères culturels significatifs dans le domaine de l'anglais juridique. D'une part, les États-Unis affichent une politique étrangère officielle de défense des droits de l'homme; d'autre part, ils se singularisent en figurant parmi les pays ayant exécuté le plus grand nombre de prisonniers en compagnie de la Chine, de l'Iran, de l'Arabie Saoudite et du Pakistan. En 2008, la Cour suprême américaine a été amenée à se prononcer sur la constitutionnalité de la peine capitale dans le cas d'un viol sur mineur. L'arrêt Kennedy v. Louisiana, particulièrement médiatisé pendant la période électorale qui opposa John McCain à Barack Obama, a suscité de nombreuses controverses dans tous les médias. Nous proposons de mettre en perspective un article de journal annonçant le recours, la transcription officielle de l'audience devant la plus haute juridiction du pays, un 
épisode d'une fiction à substrat professionnel (FASP) télévisuelle, Boston Legal, inspirée librement de cette affaire et l'arrêt de la Cour suprême.

Pour pouvoir comprendre le contexte juridique de l'application de la peine de mort, il est utile de la replacer dans une perspective historique. Ce cadrage fait l'objet de notre première partie. Après avoir défini le genre de chaque document de notre corpus d'étude, nous dressons un état des lieux des différents savoirs spécialisés présentés dans le but de déterminer lesquels de ces documents sont les mieux à même de répondre aux objectifs d'apprentissage d'une culture juridique dans une perspective linguistique. Enfin, nous consacrons notre dernière partie à l'analyse de la répartition de la terminologie juridique par texte, afin d'envisager si les documents présentés dans l'analyse peuvent être des sources lexicales efficaces servant à "acquérir une compétence à communiquer langagièrement » selon les descripteurs du CECRL (2001 : 17).

\section{Contexte historico-juridique de la peine de mort}

Utilisée pour la première fois en 1608 en Virginie, la peine capitale est encore en application dans trente-quatre États fédérés ${ }^{1}$. Lorsque, à la fin des années 1970 , se pose la question de la constitutionalité de la peine de mort ${ }^{2}$, le débat porte sur le VIII amendement de la Constitution des États-Unis. Rédigé en 1791, celui-ci fait partie de la déclaration des droits (United States Bill of Rights) qui regroupe les dix premiers amendements.

Amendment VIII: Excessive bail shall not be required, nor excessive fines imposed, nor cruel and unusual punishments inflicted.

5 L'expression "cruel punishment », utilisée pour protéger les citoyens américains contre des sanctions disproportionnées, est directement issue du Bill of Rights imposé aux souverains d'Angleterre en 1689 à la suite de la Glorious Revolution ${ }^{3}$. Celle-ci mit fin au gouvernement autocratique des Stuart au profit d'une monarchie constitutionnelle. Ce texte fondateur énonce les principes essentiels des libertés fondamentales des sujets britanniques. Les dispositions prévues par le Bill of Rights avaient pour but de mettre un terme aux supplices ainsi qu'aux peines infamantes pratiqués jusqu'au règne de l'absolutiste Jacques $\mathrm{II}^{4}$, au profit d'une justice rétributive imposant un châtiment proportionnel à la faute. Les auteurs de la Constitution américaine ont emprunté l'expression «cruel punishment » à laquelle ils y ont ajouté l'adjectif « unusual ».

Depuis la seconde moitié du vingtième siècle, un mouvement visant à restreindre les champs d'application de la peine capitale est observable dans la jurisprudence et de nombreux arrêts de la Cour suprême interprètent le huitième amendement à la lumière des évolutions des « critères de décence de la société américaine ${ }^{5}$ ». S'il n'était pas rare de condamner à mort un voleur de poules au XVII ${ }^{e}$ siècle en Virginie ${ }^{6}$, la définition des crimes les plus vils, pour lesquels la peine capitale est maintenant réservée, a évolué à travers les siècles. Dans l'affaire Kennedy v. Louisiana, la Cour suprême a évalué la fréquence à laquelle la peine capitale était prononcée pour un crime donné, afin de déterminer si la condamnation était "inhabituelle». Patrick Kennedy, le requérant dans l'arrêt Kennedy v. Louisiana, a été condamné à mort selon une législation en vigueur en Louisiane ${ }^{7}$ pour le viol d'une enfant de huit ans et l'affaire a été portée devant la plus haute juridiction fédérale le 16 avril 2008. Ses avocats ont plaidé que, sur les quelque 3300 personnes condamnées à une peine similaire, seules deux l'étaient pour des viols. 
La Cour jugea que la condamnation était inhabituelle pour l'époque, lui conférant un caractère "cruel » et a cassé la décision de la Cour suprême de la Louisiane. Elle a déclaré non conforme à la Constitution la loi autorisant la condamnation à mort pour le viol d'enfant, élargissant par là même la jurisprudence Coker ${ }^{8}$ qui avait déjà statué dans ce sens en 1977 dans le cas d'une victime adulte.

7 L'arrêt Kennedy v. Louisiana (554 U.S. 407) de 2008 a retenu notre attention en raison de sa médiatisation importante en période électorale. La condamnation controversée de Patrick Kennedy a relancé le débat sur la moralité de la justice d'élimination. Le concepteur de dispositifs pédagogiques peut donc choisir parmi de nombreux documents émis par les différents acteurs sociaux, journalistes, professionnels de la justice et créateurs de séries télévisuelles. Nous avons choisi des documents utilisés traditionnellement par les enseignants de langue et par les enseignants des champs disciplinaires (le droit ici) que nous proposons de décrire ci-dessous. Ces documents sont présentés selon la chronologie de leur parution.

\section{Analyse du corpus}

\subsection{L'article de presse (14 avril 2008)}

Le discours de la presse grand public est un genre intéressant à exploiter pédagogiquement puisque le lectorat visé n'appartient pas à la communauté professionnelle du droit. La terminologie est donc juridique, mais non hautement spécialisée. L'article de presse permet au lecteur néophyte d'accéder à des connaissances spécialisées par le biais du discours médiatisé du journaliste :

If the Supreme Court strikes down Louisiana's statute, the conviction will still stand, Jefferson Parish District Attorney Paul Connick Jr. said, as the court is only considering whether the death penalty is warranted in child rape cases. [ARTI]

L'article retenu pour cette étude a été publié dans le Times-Picayune deLa NouvelleOrléans deux jours avant l'audience qu'il annonce : «U.S. top court to hear La. child rape case. It will decide if death penalty fits the crime " [ARTI]. Il a été choisi car il présente, au même titre que la FASP, l'objet du débat mais la décision de la Cour suprême n'est pas connue. Ce déficit d'information constitue l'un des ressorts de la dynamique pédagogique.

\subsection{L'audience de plaidoirie ou oral arguments (16 avril 2008)}

Le deuxième document retenu est l'audience de plaidoirie qui a été annoncée dans le Times-Picayune. L'enregistrement se trouve sur le site Oyez dédié à l'archivage multimédia des audiences de la Cour suprême. Sur le site, il est possible de consulter le fichier audio tout en lisant la transcription écrite. Le réquisitoire et la plaidoirie durent au total une heure, chaque partie disposant d'un temps de parole prédéfini. Il apparait une différence essentielle dans le déroulement de l'audience devant la Cour suprême par rapport à une juridiction d'un degré inférieur. En effet, les différents locuteurs, juges, avocat ou procureur s'arrogent le droit de s'interrompre mutuellement comme en témoigne l'extrait suivant :

Mr. Fisher: Okay. Thank you. But-

Justice Souter: Even... even with respect to... now, I'm asking you to-

Mr. Fisher: -Right. 
Justice Souter: --to forget my question again for a minute.

Mr. Fisher: Okay. But-

Justice Souter: Even on the plurality analysis, your argument, as I understand it is, if

there is any question left in Coker, in effect it's answered by Enmund/Tison. [OYEZ]

11 Les spectateurs familiers des FASP juridiques sont habitués à des plaidoiries en forme de longs monologues, fréquents dans les procès en première instance. Il peut leur paraître incongru que, dans cet épisode, les juristes en viennent à se couper souvent la parole. La comparaison de la FASP avec la transcription officielle de la véritable audience de plaidoirie permet de vérifier que la version fictionnelle est fondée, en partie, sur une imitation de la réalité du point de vue de la forme. Sur le fond, cette source primaire fournit également la possibilité d'évaluer si la terminologie juridique présente dans la fiction est utilisée à bon escient.

\subsection{La fiction à substrat professionnel (22 avril 2008)}

L'extrait choisi provient de la série juridique Boston Legal diffusée entre 2004 et 2009 aux États-Unis et en France. L'étude porte sur un épisode diffusé six jours après la plaidoirie réelle. Trois épisodes sur les 101 qui constituent les cinq saisons abordent le thème de la peine de mort. Les scénarios sont de David E. Kelley, un avocat qui a exercé à Boston avant de se consacrer exclusivement à l'écriture et à la production de séries judiciaires 9 . Pour les épisodes abordant le problème juridique de la peine capitale, il a fait appel à Jonathan Shapiro ${ }^{10}$, ancien procureur et professeur de droit pénal à la Faculté de droit de la University of Southern California. La série se conforme donc bien aux principales conventions génériques de la fiction à substrat professionnel, ou FASP (Petit 1999), en particulier celle de l'auteur qui met son expertise professionnelle au service de l'écriture diégétique.

Chronologiquement, le premier épisode abordant le thème de la peine capitale est l'épisode 17 de la première saison diffusée en 2004. On y voit l'avocat/héros de la série, Alan Shore, se rendre au Texas pour tenter de persuader les juges de surseoir à l'exécution d'un condamné à mort.

14 L'épisode auquel s'intéresse notre analyse, The Court Supreme, paraît trois ans après. Il commence par un bref rappel de l'entrevue entre le juge texan et l'avocat de la défense :

Judge Christopher Serra: Mr. Shore, you came down here from Massachusetts?

Alan Shore: Yes.

Judge Christopher Serra: You know what I'd like to propose? I'd like to propose that you got a problem with the death penalty in general. Now is that why you came here, sir? [FASP]

L'extrait introduit par la formule consacrée «Previously on Boston Legal »vise à renseigner le téléspectateur qui aurait pris la série en cours sur la posture idéologique de la direction artistique. Ce rappel découle de la nécessité de répéter le trait essentiel de la quête du héros : faire prévaloir les droits de l'homme et rappeler les obstacles qui se dressent sur son chemin.

Nous avons retenu dans le cadre de notre analyse toutes les scènes concernant le thème de la peine capitale, laissant de côté les séquences relatives à l'arc saisonnier ${ }^{11}$, une histoire d'amour entre l'un des avocats du cabinet et une prostituée. Dans cet épisode, l'avocat est sollicité pour plaider devant la Cour suprême des États-Unis et défendre le cas de Leonard Serra, un noir américain condamné à mort pour le viol d'une fillette de 
huit ans dans l'État de la Louisiane. Le parallèle avec l'affaire réelle qui a été plaidée la semaine précédente est assez manifeste.

Si le fond de l'affaire est similaire à la réalité, l'univers diégétique comporte des éléments fictionnels puisque l'avocat/héros n'a que deux jours pour préparer sa défense. Il consacre le peu de temps qui lui est imparti à rendre visite à son client et à s'entraîner devant une équipe spécialisée dans les plaidoiries à la Cour suprême. La dimension pédagogique de cette scène est d'une grande valeur pour l'enseignement de la culture de spécialité puisque le protagoniste se trouve dans la position d'un apprenant qui acquiert des compétences culturelles et pragmatiques afin de pouvoir réaliser une tâche :

You need five votes. You're not gonna get Roberts, Scalia, Thomas or Alito, so, don't even bother. You should get Ginsburg, Breyer, Souter and Stevens. Kennedy ${ }^{12}$ is the swing. You need to focus on Kennedy. [...] Don't waste your time introducing yourself, they'll know who you are. [...] If you speak with a tone like that, you're done! [FASP]

18 La structure narrative de la série Boston Legal est quasiment immuable. Chacun des 101 épisodes se décompose en trois phases: «nouement, climax et dénouement » selon la terminologie utilisée par Vincent Colonna (2010: 278). La structure de The Court Supreme est différente de tous les autres épisodes car le spectateur est confronté à « un schéma actanciel inachevé » (Couprie citant Greimas 1995 : 21) car il ne connaît pas, à la fin de l'épisode, diffusé le 22 avril 2008, la décision qui sera prise par les neuf juges de la Cour suprême le 25 juin 2008.

Cette structure narrative, qui rompt avec l'architecture traditionnelle de la série, agit comme un miroir de la réalité car il faudra encore deux mois au condamné pour savoir s'il va être exécuté ou si sa sentence va être commuée en peine d'emprisonnement à perpétuité. Le spectateur vit par procuration les émotions du condamné et l'empathie créée avec ce personnage suscite une curiosité qui ne sera satisfaite qu'en suivant l'actualité juridique. Le concepteur de dispositif pédagogique peut utiliser les ressorts de la fiction pour faire émerger l'envie d'accéder à l'information par le biais du texte. Le document professionnel ou un article de presse présentant les conclusions des juges peut alors être « investi comme un objet de désir » pour paraphraser Philippe Meirieu $(2000: 2)$.

\subsection{L'arrêt (25 juin 2008)}

La décision attendue par le condamné et le spectateur est l'arrêt de la Cour suprême rendu le 25 juin 2008. C'est un document de 65 pages qui se décompose en trois parties. Le syllabus de cinq pages est un résumé sans valeur juridique rédigé par les greffiers. La partie suivante, de 36 pages, exprime l'opinion majoritaire de la Cour ( $«$ the concurring opinion ») et se conclut par la formule exécutoire : «The judgment of the Supreme Court of Louisiana upholding the capital sentence is reversed. [...] It is so ordered » [RULE].

21 La troisième partie, de 24 pages, exprime l'opinion dissidente (" the dissenting opinion ») et n'est pas un acte de langage constitutif. Elle a uniquement une valeur informative. Rédigée par le Juge Alito, elle se termine par un commentaire sur la relation prédicative, porté par la modalité en would:

The party attacking the constitutionality of a state statute bears the "heavy burden" of establishing that the law is unconstitutional. [...] That burden has not 
been discharged here, andI would therefore affirm the decision of the Louisiana

Supreme Court. [RULE] événement à venir pour l'article de presse sélectionné, participer à l'élaboration d'un acte juridictionnel pour la plaidoirie, sensibiliser les téléspectateurs aux droits de l'homme tout en les distrayant pour la FASP, faire jurisprudence pour l'arrêt. Étant donné que ces documents diffèrent en genre et en taille, scénographie judiciaire d'un côté, discours de professionnel de l'autre, certains savoirs spécialisés et certains termes se retrouveront dans les quatre textes alors que d'autres ne sont présents que dans un genre particulier.

\section{Comparaison des savoirs spécialisés}

\subsection{Points communs}

Les quatre textes présentent l'affaire et les opinions divergentes relatives au caractère constitutionnel de la loi en Louisiane. On note aussi que tous les documents rendent compte de la posture singulière de l'État de la Louisiane qui fait partie de la petite minorité des États ( 5 sur 35 à l'époque) à autoriser la peine capitale pour un viol n'ayant pas entraîné la mort de la victime («non homicide rape»). Chaque texte mentionne la jurisprudence de 1977 qui concerne le viol d'un adulte, comme dans cet exemple :

Alan Shore: This court already held in Coker v. Georgia that the death penalty was not authorized in non homicide rape.

Justice: That case did not speak to the rape of a child. [FASP]

Enfin, on trouve des références à la Constitution dans tous les documents, comme dans cet extrait : "Kennedy's attorneys argue the law is contrary to the Eighth Amendment's cruel and unusual punishment clause » [ARTI].

Les éléments juridiques essentiels sont donc présents dans les quatre textes. La différence majeure réside dans le degré d'implicite inhérent à leur genre. L'audience de plaidoirie étant un débat entre experts, le contexte est implicite à la discussion comme en témoigne la première phrase prononcée par l'avocat de Kennedy : "Mr. Chief Justice and may it please the Court: This country has not executed anyone for a rape of any kind in over 43 years » [OYEZ]. L'enseignant désireuxde didactiser ce support devra donc l'étayer par de nombreuses références extratextuelles.

En revanche, la FASP, qui met en scène des affaires judiciaires différentes à chaque épisode, a recours à une scène d'exposition synthétique qui fait l'économie de cette mise en contexte : "We've got a new client, he raped an eight-year old, [...]. The man has been sentenced to death» [FASP]. Ainsi, du fait de leur genre différent, certains savoirs spécialisés ne sont pas présents dans tous les textes comme nous le mettons en évidence dans la section suivante. 


\subsection{Contenus différents}

28 dans une analyse détaillée. En effet, $10 \%$ du texte sont composés de citations des arrêts précédents. Si les autres documents mentionnent tous l'arrêt Coker, le document émanant de la Cour suprême fait état de 46 jurisprudences différentes relatives au sujet. Par ailleurs, le descriptif des préjudices physiques subis par la victime n'est exposé que dans les documents professionnels de manière très détaillée.

A contrario, seule la FASP présente le fonctionnement de la Cour suprême grâce à la scène où l'avocat fictif prend un cours sur la manière de plaider devant cette juridiction :

You need five votes. [...] Keep your argument focused on constitutional issues. Do not - do not - do not be talking about your client. [FASP]

De même, seule la FASP offre un état des lieux des écarts entre les dispositions constitutionnelles, qui prévoient un traitement égal des personnes (« equal protection of the laws $\left.{ }^{13} »\right)$ et la réalité :

Alan Shore: In Louisiana, historically, it's been blacks that have been executed for rape in non homicide cases. In the last hundred years, Louisiana has executed 29 men for rape. All were black. [FASP]

Enfin, le " genre sériel » selon les termes de Jean-Pierre Esquenazi (2009:13), grâce à sa dimension visuelle, donne un aperçu du protocole puisqu'une séquence montre un des avocats plaider en queue de pie comme le veut la tradition ${ }^{14}$. L'épisode présente des éléments de "mimésis formelle " (Schaeffer 1999: 140) puisque la diégèse permet la rencontre entre le personnage fictif et des juges incarnés par des acteurs ressemblant trait pour trait aux personnages réels qui se trouvent fonctionnalisés :

C'est que la stratégie de contamination la plus communément mise au service de l'effet de réel - et réalisée de manière exemplaire par la fiction réaliste - consiste à introduire des éléments référentiels - historiques, mais aussi géographiques, temporels, etc. - dans l'univers inventé. (Schaeffer 1999 : 142)

Dans une perspective didactique, cette dimension visuelle apporte une aide considérable à la compréhension car les arguments juridiques sont avancés par des personnages immédiatement reconnaissables par la sémiotique visuelle. Hugo Bowles propose une analyse clarifiant les raisons pour lesquelles les Newspaper law reports sont d'accès difficiles ; elles s'appliquent également à l'arrêt :

Frequently, a combination of intratextual cross-referencing and extra-textual inferencing is required to understand certain conventions. [...] [I]t requires greater powers of inference [...] if the reader is to understand which side of the dispute the argument is coming from. (1995:219)

Pour un apprenant, les dénominations utilisées dans l'arrêt, "demandeur » ou " appelant», «défenseur» ou "intimé» par exemple, peuvent compliquer le traitement simultané de tous les éléments d'information comme il apparaît dans les exemples suivants : " [T]he Constitution bars respondent from imposing the death penalty [...]. The court acknowledged that petitioner would be the first person executed [...] » [RULE]. Ce risque de surcharge cognitive est moins important dans la FASP qui privilégie le dialogue, l'utilisation des pronoms personnels plutôt que le style indirect, procédés qui facilitent considérablement l'accès au sens : «I [the lawyer] have a problem with the State executing a man with diminished capacity " [FASP]. 
34 Même si la fiction est généralement fondée sur un contrat narratif implicite d'illusion réaliste, les créateurs se permettent de déroger à cette convention pour dresser un inventaire des limites du système judiciaire. Dans une digression que seul l'univers diégétique autorise, l'avocat fictif profite de la plaidoirie pour adresser aux juges des critiques concernant le système d'élection des magistrats qui incite à la pratique d'une politique répressive, "tough on crime ", à des fins électoralistes. L'avocat suggère par ailleurs, que l'intégrité de certains juges est sujette à caution :

Alan Shore: Justice Alito, you were caught hearing a case involving a company you'd invested hundreds of thousands of dollars in. Huh. No conflict of interest there? You also don't recuse yourself in terrorism cases even though your best friend is Michael Chertoff, head of homeland security. [FASP]

Le privilège de l'auteur de fiction est de profiter de toutes les libertés du créateur et de modifier la réalité à sa guise à des fins de divertissement ou à des fins didactiques. La fiction est ici au service de la culture spécialisée parce que les personnages sont des figures rhétoriques qui dépassent le cas traité dans les documents professionnels et l'article du Times-Picayune. Le condamné fictif est une construction métaphorique intéressante à déconstruire. Les auteurs ont créé un personnage polysémique dans le but de montrer que la société américaine empêche parfois l'exercice des libertés fondamentales. À la fois bourreau et victime d'un système fondé sur la vengeance, le condamné incarne toutes les injustices dénoncées par les abolitionnistes ${ }^{15}$ puisqu'il est à la fois noir, juridiquement irresponsable et qu'il clame son innocence :

Alan Shore: Did he do it?

Defendant's lawyer: He says he didn't. He has an IQ of 70, so-

Alan Shore: Well, executions of the mentally disabled are unconstitutional, so-

Defendant's lawyer: He was never officially pronounced disabled. A 70 IQ only puts

him in range, but in Louisiana-

Alan Shore: Oh, he could be governor! [FASP]

On note que la réplique incisive de l'avocat/héros est le miroir de l'opinion des intellectuels américains qui se positionnent en défenseurs des droits de l'homme. À l'instar de John Grisham qui s'est engagé à travers l'écriture de romans FASP $^{16}$ et qui use de sa renommée artistique pour servir la cause des abolitionnistes ${ }^{17}$, David E. Kelley réalise une métonymie textuelle de l'Amérique qui veut abolir la peine de mort. La force de la FASP télévisuelle réside dans la richesse de la dimension théâtrale qui ne peut se réduire au seul vocabulaire. La gestuelle, le décor sonore, les effets de voix, tout le langage paraverbal fait sens et soutient le texte. Ces caractéristiques en font un support d'enseignement particulièrement riche sur le plan des savoirs. Si les informations concernant l'injustice de la peine d'élimination ( We have an epidemic of wrongful executions " [FASP]), la discrimination raciale ainsi que la corruption des juges font l'objet d'articles de fond dans certains journaux, aucun de ces sujets n'est, à notre connaissance, abordé dans les articles relatifs à l'affaire Kennedy. Ces observations sont d'autant plus surprenantes que les quatre documents n'obéissent pas aux mêmes contraintes d'écriture. L'épisode de Boston Legal doit suivre le format de 42 minutes imposé par la chaîne de production. C'est pourtant la source offrant le plus d'informations générales concernant le thème de la peine de mort pour un récepteur souhaitant développer des connaissances sur le sujet. Nous pensons par exemple aux étudiants inscrits en master 2 " Théorie et pratique des droits de l'homme » à la faculté de Grenoble dont l'objectif institutionnel requiert une « exigence de théorisation et de connaissances dans le domaine des grands défis contemporains concernant la protection et la promotion des droits de l'homme ${ }^{18}$ ». Il reste à définir si le document 
peut servir aussi à élargir la terminologie juridique des étudiants et si celle-ci est attestée par les sources primaires.

\section{Comparaison de l'utilisation de la terminologie juridique}

\subsection{Méthodologie de constitution du corpus lexical}

Notre posture est celle de l'enseignant d'anglais de spécialité qui cherche à enrichir la compétence linguistique des étudiants tout en développant leur culture juridique. Par expérience, nous savons que la majorité des étudiants de droit, même ceux qui ont atteint la $5^{\mathrm{e}}$ année, ne savent pas comment dire en anglais " requérir la peine de mort " (" to pursue the death penalty ») ou même "le tribunal " (" court house »). Le corpus est donc composé du lexique nécessaire pour s'exprimer dans le domaine du droit pénal (« to face sentencing », « to rule », par exemple) et de la procédure législative (« to pass a law ", «to come into effect», etc.). Par ailleurs nous avons relevé dans les textes les expressions pouvant faire barrage à la compréhension (« to capitalize an offense »). Aussi la définition de "terminologie juridique " recouvre-t-elle, dans cette étude, une acception large. L'ensemble est composé de termes hautement spécialisés mais il comporte également une intersection avec la langue dite générale selon la définition proposée par Catherine Resche :

[o]n concevra qu'il est difficile, voire impossible, de déterminer avec certitude où situer la frontière et on parlera plus volontiers de continuum. Or, précisément, si continuum il $\mathrm{y}$ a, cela sous-entend un espace flou où l'on quitte progressivement le territoire de la langue générale pour pénétrer plus avant dans le domaine de la langue spécialisée. (2001:37)

Nous avons, par exemple, relevé les occurrences des adjectifs «cruel and unusual » qui sont souvent cités dans les FASP relatives à la peine capitale. On trouve notamment, dans l'épisode que cette étude concerne, la réplique "cruel and unusual hypocrisy». La référence implicite à la Constitution n'a aucun sens pour un étudiant qui ignore le huitième amendement. Jean-Louis Trouillon souligne qu'une étude lexicale décorrélée de la culture véhiculée par la langue de spécialité n'a pas de sens :

Une langue de spécialité ne relève donc pas seulement de données terminologiques propres à un domaine donné mais d'un vaste ensemble regroupant, outre les aspects purement linguistiques (terminologie, grammaire, style, etc.), la culture des milieux qui utilisent cette langue. $(2010: 26)$

Les termes retenus dans le cadre de cette analyse peuvent donc être répartis en trois catégories : les termes strictement juridiques comme «jury trial » ou « legal action», des termes hautement polysémiques qui sont identifiés comme appartenant à la catégorie sémantique du droit grâce au contexte : "a sentence ", "This court already held in Coker $[. .$.$] » et des collocations : " capital punishment », « death row », etc. Un concordancier { }^{19}$ a ensuite été utilisé pour déterminer leur présence dans chaque document. Les termes sont donnés en annexe et chaque item ou phraséologie relevant du domaine du droit regroupe tous les mots de la même famille. C'est ainsi, par exemple, que le lemme noté " convict " (verbe et substantif) comprend aussi toutes les formes dérivées (« convicted», «conviction»). 
Nous sommes consciente qu'en comparant des transcriptions de dialogues et des documents rédigés dans un style écrit, nous nous heurtons à un problème de comptage des mots puisque l'oral favorise les formes contractées comme dans ces exemples: "they're gonna kill him ", "you got a problem with the death penalty " [FASP]. Le segment " gonna » compte pour un mot alors que dans l'extrait de l'arrêt on trouve des occurrences de "going to ${ }^{20}$ » qui comptent pour deux mots. Les chiffres annoncés ne tiennent donc pas compte de ces spécificités. Néanmoins, comme on peut l'observer dans le graphique qui rend compte de la totalité des mots de chaque document, les écarts entre les textes sont suffisamment élevés pour que les spécificités liées à la nature des textes ne corrompent pas les résultats de manière significative.

\subsection{Analyse de la terminologie}

On remarque dans la figure 1 que l'arrêt comporte vingt fois plus de mots que l'article de journal (20 924 contre 1072 mots) et que l'on dénombre quatre fois plus de mots dans la plaidoirie que dans la FASP (11 653 contre 3 310).

Figure 1. Nombre de mots par documents

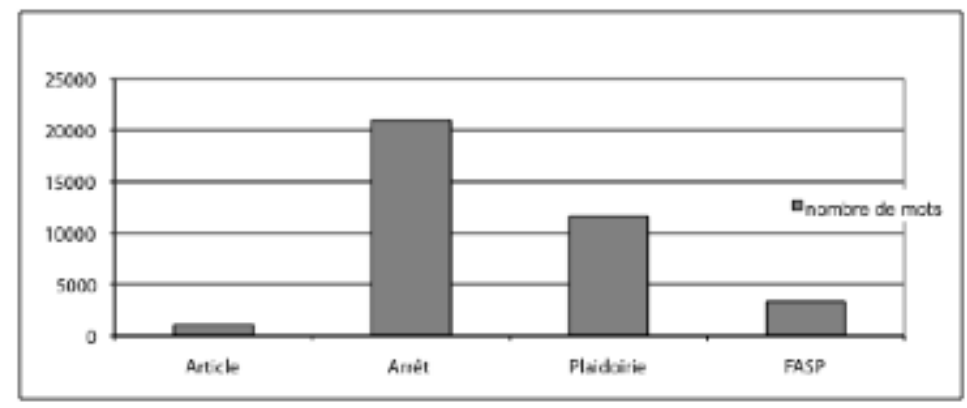

Si l'on compare ces données à l'aune du pourcentage de termes juridiques rencontrés dans chaque document (figure 2), on observe que, sur 168 termes repérés en tout, $77 \%$ sont présents dans l'arrêt, $49 \%$ dans la plaidoirie orale, $47 \%$ dans la FASP et $30 \%$ dans l'article de journal. La FASP, qui comporte six fois moins de mots que l'arrêt, possède près de la moitié du lexique. Il n'y a donc que trente points de différFremarquonsence dans un document six fois plus court. On constate ainsi que l'épisode de la série juridique est une ressource lexicale aussi riche que la plaidoirie authentique qu'elle imite, alors qu'il possède quatre fois moins de mots. Il n'est pas surprenant de trouver une plus grande diversité de termes juridiques dans les textes professionnels. Cet écart peut s'expliquer par le genre et la fonction de chaque document comme nous allons tenter de le montrer par quelques exemples. 
Figure 2. Pourcentage de termes juridiques

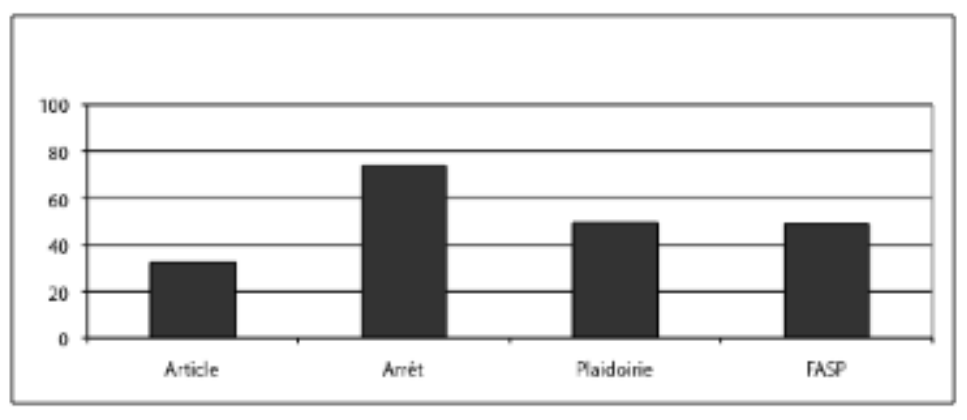

\subsubsection{Lexique particulier} burglary » dans un arrêt concernant la dimension constitutionnelle de la peine de mort en cas de viol. Ces infractions sont mentionnées comme exemples de circonstances aggravantes ("aggravating circumstances») nécessaires pour pouvoir requérir la peine de mort dans le cas d'un homicide. Ces termes sont absents de la FASP et de l'article de presse. On observe néanmoins que les documents professionnels et la fiction font mention de crimes contre les intérêts de la nation pour lesquels la peine de mort est applicable au niveau fédéral : "We also have the death penalty for air piracy and treason, also non homicide offenses » [FASP].

La FASP ayant pour objectif de dénoncer les violations des droits de l'homme, la phraséologie privilégiée est celle des entraves aux libertés fondamentales. Aussi trouve-t-on des expressions telles que « to botch executions » ou « a man's life is at stake ». $\mathrm{Si}$ les juges, les avocats et les journalistes, emploient exclusivement l'expression « to 
execute a convict », les scénaristes utilisent par sept fois le verbe " to kill » pour exprimer la même idée. Les documents authentiques n'appliquent ce verbe qu'aux crimes. La rhétorique de la FASP est similaire à celles des associations de défense des droits de l'homme qui parlent de «meurtre commis par l'État». Par sa fonction humaniste, la FASP est plus centrée sur le condamné alors que les autres documents sont focalisés sur la victime.

L'intention de communication est donc perceptible dans le genre discursif et chaque registre terminologique : cet article de la presse informe, la plaidoirie convainc des professionnels, la FASP éduque et divertit, et l'arrêt pose un acte juridictionnel. L'enseignant d'anglais de spécialité devra évaluer à quel récepteur ses étudiants s'apparentent le plus pour déterminer son choix de supports pédagogiques en fonction de la situation de communication qu'il vise, comme l'affirmaient Tom Hutchinson et Alan Waters:

A text alone has no value. A text is a message from a writer to an assumed reader. In writing the text the writer will make a judgement as to the knowledge the assumed reader will bring to the text and the use the reader will make of it. $(1987: 159)$

\subsubsection{Lexique commun}

Les spécificités de chaque document favorisent certains champs sémantiques. Néanmoins, outre celles-ci, nous remarquons que la phraséologie juridique repérée dans l'article de presse et la FASP est corroborée par les documents professionnels. En ce qui concerne les règles d'adresse, l'oralité est propice à leur utilisation puisqu'elles expriment les façons de s'adresser à un juge ("Justice Thomas »), à un avocat ("Counsel » ), etc. La comparaison lexicale entre représentation fictionnelle et réalité permet de vérifier que les "unités phraséologiques" (Gledhill 2011:10) utilisées dans le genre sériel sont authentiques, notamment les expressions comme "May it please the Court " ou encore « Mr Chief Justice».

On observe aussi que la terminologie relative au rôle des juridictions d'appel est attestée par les documents professionnels. Les enseignants de langue pourront de ce fait utiliser la FASP comme ressource lexicale pré-requise pour la compréhension d'une décision de justice. En effet, la fiction et l'arrêt sont porteurs d'expressions telles que " casser une décision de justice » (« to reverse », « to overturn a decision »), « invalider une loi » (« to strike down a law»). Ce lexique est donc utilisé à bon escient par les auteurs de la FASP, ce qui n'est pas très surprenant du fait de leur expertise professionnelle.

51 Les observations précédentes sont aussi vérifiables pour le lexique relatif à la responsabilité pénale d'un accusé. Les termes "diminished capacity ", «IQ below 70 », et les concepts qui s'y rapportent, sont présents dans les documents professionnels et la FASP. L'évaluation des causes d'irresponsabilité pénale n'étant pas en France fondée sur le quotient intellectuel contrairement aux États-Unis, la différence culturelle peut faire l'objet de débat dans lequel les étudiants peuvent faire appel à leur expertise en matière juridique pour alimenter la discussion en langue cible.

\section{Conclusion}

52 L'étude de la peine capitale en cours d'anglais juridique permet de rappeler que les droits de l'homme n'ont pas de fondement transculturel puisqu'ils ne se déclinent pas 
de la même manière à Paris, Texas et à Paris, capitale de la France. L'objectif principal du cours de langue est d'accroître la motivation intrinsèque des apprenants en choisissant des supports d'enseignement qui stimulent l'envie d'être acteur de son apprentissage culturel. L'atout majeur des fictions inspirées de faits réels réside dans l'envie qu'elles déclenchent chez le spectateur de vérifier quels sont les éléments qui relèvent de la réalité.

Il peut paraitre réducteur de tenter de trouver des points communs entre une comédie télévisuelle, un article de presse, une audience devant des juges et un arrêt de la Cour suprême puisque ces documents appartiennent à des genres fondamentalement différents. Néanmoins, l'analyse des textes a montré que la fiction respecte le schéma formel de la véritable audience de plaidoirie, que l'oralité facilite la compréhension des concepts juridiques, que, malgré la contrainte temporelle, la FASP est riche en savoir spécialisé et que, d'un point de vue didactique, le format de quarante-deux minutes peut être un véritable atout. Enfin, l'analyse a montré qu'une grande partie de la terminologie est commune aux sources primaires du droit, conférant aux documents non professionnels un caractère authentique utilisable à des fins pédagogiques. Cette démonstration vient confirmer l'affirmation de Shaeda Isani selon laquelle :
À l'opposé du document authentique, qui se limite nécessairement au registre normé qui lui est spécifique, l'un des principaux atouts didactiques de la FASP est sans doute qu'elle offre un reflet fidèle de la diversité discursive née de l'existence de multiples sphères d'interaction au sein d'une même communauté professionnelle. (2010:112)

Le message véhiculé par le genre sériel est éminemment didactique et nous rappelons à cet égard que l'un des auteurs de la FASP est un professeur de droit pénal qui vise à faire connaitre le fonctionnement du système judiciaire et à donner une conscience politique au téléspectateur. La FASP peut être, dans ce contexte, un support d'enseignement tout particulièrement adapté pour initier les apprenants aux questions qui préoccupent la communauté professionnelle du droit à l'échelle mondiale.

Par ailleurs, la faible part de l'oral face à la place très importante de l'écrit dans le cursus de l'étudiant de droit nous amène à penser que l'oral doit retrouver ses lettres de noblesse. À condition de s'assurer que les éléments de fictionnalité présents dans la comédie soient bien identifiés comme tels, l'exploitation didactique de la FASP peut aussi servir à exposer les étudiants à la langue orale et à répondre à un besoin de dynamisme sensoriel.

D'aucuns verront la comédie comme un barrage à la transmission fiable d'une culture juridique puisqu'il est impossible pour un avocat de tancer un Juge de la Cour suprême lors d'une audience, comme c'est le cas dans l'épisode de Boston Legal. Il serait donc intéressant de poursuivre cette étude en nous interrogeant sur la capacité des apprenants à identifier les marqueurs de fictionnalité et à envisager les moyens de médiation à la disposition de l'enseignant pour « remédier à la perspective limitée, voire erronée, du milieu spécialisé présenté par le récit fictionnel et, au-delà, pour éveiller les facultés critiques de l'apprenant » (Isani 2010 : 121).

Nous remercions Madame Florence Bouvier, Vice-procureur chargée des mineurs et de la famille au Tribunal de grande instance de Grenoble pour la relecture minutieuse de la terminologie juridique française utilisée dans cet article ainsi que Monsieur Fabien Girard, maître de conférences en droit privé à la faculté de droit de Grenoble pour ses éclairages sur la procédure pénale américaine. 


\section{BIBLIOGRAPHIE}

\section{Sources primaires}

Boston Legal, Saison 04, Episode 17. Consulté le 28 avril 2010. <http://www.tvsubtitles.net/ subtitle-12702.html>. [FASP]

Kennedy v. Louisiana. ( $\left.\mathrm{N}^{\circ} 07-343\right)$ Oral arguments. Consulté le 15 novembre $2010<\mathrm{http}: / /$ www.oyez.org/cases/2000-2009/2007/2007_07_343/argument/>. [OYEZ]

Kennedy v. Louisiana, 554 U.S. 407 (2008). Consulté le 28 décembre 2010. <http:// www.scotusblog.com/wp-content/uploads/2008/06/07-343.pdf>. [RULE]

Purpura, Paul. 2008. « U.S. top court to hear La. child rape case. It will decide if death penalty fits the crime ». Times Picayune. Consulté le 15 décembre 2010 <http://www.nola.com/news/ index.ssf/2008/04/us_top_court_to_hear_la_child.html>. [ARTI]

\section{Références}

Bowles, Hugo. 1995. « Why are newspaper law reports so hard to understand? ». English for Specific Purposes 14/3, 201-222.

Colonna, Vincent. 2010. L'art des séries télé. Paris : Payot \& Rivages.

Conseil de L'Europe. 2001. CECR. Paris : Éditions Didier.

Couprie, Alain. 1995. Le théâtre. Paris : Nathan.

Esquenazi, Jean-Pierre. 2009. Mythologie des séries télé. Paris : Le cavalier bleu.

Gledhill, Christopher. 2011. « The lexicogrammar approach to analysing phraseology and collocations in ESP texts ». ASp 59, 5-23.

Greenstein, Rosalind. 2005. « Du vouloir dire au vouloir faire : le cas de l'anglais du droit ». In Greenstein, Rosalind (dir.), La langue, le discours et la culture en anglais du droit. Paris : Publications de la Sorbonne, 125-152.

Hutchinson Tom \& Alan Waters. 1987. English for Specific Purposes, a learning-centred approach. Cambridge : Cambridge University Press.

Isani, Shaeda. 2010. «Dynamique spéculaire de la fiction à substrat professionnel et didactique des langues de spécialité ». ASp 58, 105-123.

Meirieu, Philippe. 2000. «L'art dans l'éducation, poudre aux yeux ou discipline fondamentale?». Association pour la promotion et l'enseignement des arts visuels. Consulté le 16 avril 2011. <http://www.apeav.ch/documents/medias/philippe_meirieu_conf.pdf>.

Petit, Michel. 1999. « La fiction à substrat professionnel : une autre voie d'accès à l'anglais de spécialité ». ASp 23-26, 57-81.

Resche, Catherine. 2001. « Réflexions sur la frontière entre langue générale et langue spécialisée ». In Mémet Monique \& Michel Petit (dir.), L'anglais de spécialité en France, Mélanges en l'honneur de Michel Perrin. Bordeaux : GERAS-Éditeur Université Bordeaux 2, 37-46.

Schaeffer, Jean-Marie. 1999. Pourquoi la fiction? Paris : Édition du Seuil.

Swales, John. 1990. Genre analysis. English in academic and research settings. Cambridge : Cambridge University Press. 
Trouillon, Jean-Louis. 2010. Approches de l'anglais de spécialité. Perpignan : Presses

Universitaires de Perpignan.

\section{NOTES}

1. À la date du 9 mars 2011

2. Furman v. Georgia 408 U.S. 238 (1972)

3. $1688-1689$

4. James II of England

5. Trop v. Dulles, 356 U.S. 86,101 (1956)

6. Divine, Moral and Martial Laws, 1612

7. La. Stat. Ann. § $14: 42$ (West 1997 and Supp. 1998)

8. Coker v. Georgia, 433 U.S. 584 (1977)

9. <http://www.imdb.com/name/nm0005082/> consulté le 12 juin 2011

10. <http://www.imdb.com/name/nm1236705/> consulté le 12 juin 2011

11. Histoire qui lie les personnages d'un épisode à l'autre.

12. Anthony M. Kennedy, juge à la Cour suprême depuis 1988, et non Patrick Kennedy le requérant dans la jurisprudence Kennedy v. Louisiana.

13. Amendement 14 de la Constitution américaine

14. Les plaidoiries devant la Cour suprême ne sont jamais filmées contrairement à certains procès en première instance.

15. <http://www.deathpenaltyinfo.org> consulté le 15122011

16. A time to kill 1989, The chamber 1994, The confession 2010

17. Nous pensons à la tentative d'empêcher l'exécution de Teresa Lewis en Virginie en septembre $2010 \quad<\mathrm{http} / /$ www.washingtonpost.com/wp-dyn/content/article/2010/09/10/ AR2010091002673.ht ml> consulté le 17022011.

18. $\quad<\mathrm{http}$ //webu2.upmf-grenoble.fr/facdroit/formations/

formations_m2_theorie_pratique_droits_hom me.php> consulté le 20 juin 2011

19. KWIC version 5.0

20. «I'm going to tell the same story », propos enregistrés de la victime, retranscrits dans l'arrêt.

\section{RÉSUMÉS}

Dans une perspective didactique, cet article présente une analyse contrastive de quatre documents relatifs à une affaire qui a fait date dans les annales juridiques américaines : l'arrêt Kennedy v. Louisiana dans lequel il est déclaré que la peine de mort est non conforme à la Constitution des États-Unis dans le cas d'un viol sur enfant n'ayant pas entraîné la mort de la victime. Nous analysons un article de presse, un épisode de série télévisuelle, la transcription officielle des plaidoiries devant les juges de la Cour suprême et l'arrêt qui s'y rapporte afin de comparer les savoirs spécialisés et la terminologie juridique présente dans chaque document. Cette approche quantitative cherche à déterminer si les documents comme la fiction télévisuelle à substrat professionnel et l'article de presse peuvent constituer des vecteurs pédagogiques du 
spécialisé suffisamment riches et complets sur le plan lexical et culturel pour des étudiants du secteur LANSAD.

In the didactic perspective of English for legal purposes, this paper seeks to present an analysis of four documents related to the landmark Supreme Court ruling Kennedy v. Louisiana which states that the death penalty is unconstitutional in non homicide child rape convictions. We analyse a newspaper article, one episode of a popular TV series, the transcript of the oral arguments before the Supreme Court Justices and the ruling to compare the specialised content and the legal terminology. In an essentially quantitative approach, we seek to determine whether texts such as popular legal fiction and newspaper articles are satisfactory pedagogic tools in the acquisition of the language and culture of legal English for law students.

\section{INDEX}

Keywords : English for legal purposes, legal English, LSP didactics, newspaper article, professionally-based fiction, specialised discourse, Supreme Court ruling

Mots-clés : anglais juridique, arrêt de la Cour suprême des États-Unis, article de presse, didactique des langues de spécialité, discours spécialisé, fiction à substrat professionnel

\section{AUTEUR}

\section{SANDRINE CHAPON}

Sandrine Chapon est PRCE à la Faculté de droit de Grenoble et doctorante à l'Université Stendhal Grenoble 3, sous la direction de Shaeda Isani. Sa recherche porte sur les séries juridiques américaines comme support d'enseignement. sandrine.chapon@upmf-grenoble.fr 